\title{
Computed Tomography Thorax- Role in the Diagnosis of Pulmonary Tuberculosis
}

\author{
RK. Jayshree Devi' ${ }^{1}$ Kh. Mani Singh ${ }^{2}$ \\ ${ }^{1}$ Department of Radiodiagnosis, Jawaharlal Nehru Institute of Medical Sciences, Porompat, Imphal, Manipur, India. \\ ${ }^{2}$ Department of Radiodiagnosis, Jawaharlal Nehru Institute of Medical Sciences, Porompat, Imphal, Manipur, India.
}

\section{ABSTRACT}

\section{BACKGROUND}

Tuberculosis is one of the leading causes of mortality in a developing country like India among the infectious diseases. Early diagnosis of tuberculosis is by chestX-Ray, sputum examination, culture and nucleic acid amplification. Chest X-Ray findings are not very specific, sputum examination and culture take many days thus delaying the diagnosis. CT scan is very fast, non-invasive and accurate. It can diagnose the cause of unexplained cough, chest pain, fever and other chest symptoms. Because of accuracy, high speed and better resolution, CT scan is the modality of choice for early detection of pulmonary TB. The aim of the study is to evaluate the role of CT and HRCT in pulmonary TB.

\section{METHODS}

It is a cross sectional study conducted at Jawaharlal Nehru Institute of Medical Sciences from January 2016 to June 2016 in a series of cases sent for CT or HRCT using AQUILION, Toshiba 64 slice multi detector CT. All the data were entered in excel sheet and transferred to SPSS Version 2 and then analysed by using descriptive statistics like mean, standard deviation and percentage. Chi square test was used. $p$ Value of $<0.05$ was taken as statistically significant.

\section{RESULTS}

Among 358 cases, 49.7\% (178 cases) were diagnosed as pulmonary tuberculosis. In cases diagnosed as pulmonary TB, $104(58.4 \%)$ were male and $74(41.7 \%)$ were female. Over 60 yrs. of age, $56.1 \%$ of the cases had TB with cavitary and parenchymal lesion, followed by TB with lung destruction \& cicatrisation in $17.3 \%$ of the cases. In younger age group, 20 years and below, had TB with cavitary \& parenchymal lesions in $75 \%$ of cases and TB with chronic empyema \& fibrothorax in $25 \%$ cases. Incidence of TB in higher age groups was statistically significant $(\mathrm{p}<0.05)$.

\section{CONCLUSIONS}

CT and HRCT can diagnose different stages of pulmonary TB and any associated diseases. It helps in taking treatment decisions and preventing disease spread which is an important health issue. For early detection of pulmonary TB, CT and HRCT is a must for diagnosis.

\section{KEY WORDS}

Tuberculosis (TB), Computed Tomography (CT), HRCT, Cough, Cavitary Lesions, Consolidation
Corresponding Author:

Dr. Kh. Mani Singh,

Sagolband Tera Bazar,

Sapam Leirak, Imphal West-795001,

Manipur, India.

E-mail: khmani123singh@gmail.com

DOI: $10.14260 /$ jemds/2020/96

Financial or Other Competing Interests: None.

How to Cite This Article:

Devi RKJ, Singh KHM. Computed tomography thorax-role in the diagnosis of pulmonary tuberculosis. J. Evolution Med. Dent. Sci. 2020;9(07):422-427, DOI: 10.14260/jemds/2020/96

Submission 14-12-2019,

Peer Review 22-01-2020,

Acceptance 29-01-2020,

Published 17-02-2020.

\section{(i)}




\section{BACKGROUND}

Tuberculosis (TB) is a common health concern causing high mortality and morbidity for both developing and developed countries. ${ }^{1}$ In developing country like India, pulmonary tuberculosis is widespread among the old people and poor socio-economic class. TB can mimic pneumonia, malignancy and interstitial lung diseases (ILD) and sputum smear examination takes time for diagnosis. ${ }^{2}$ The goal of National Strategic Plan (NSP) 2017-2025 for TB elimination is to achieve a rapid decline in burden of $\mathrm{TB}$, morbidity and mortality while working towards elimination of TB in India by 2025. It infects one third of world population, thereby making the disease a major public health issue. Among the infectious diseases it is a main killer. In our country it is one of the leading causes of mortality. ${ }^{3}$ It is common disease in socio economically disadvantaged, elderly and chronic debilitated individuals. ${ }^{3}$ Risk of developing active TB depend on immune status, extremes of age, malnutrition, cancer, immunosuppressive therapy, HIV infection, end stage renal disease and diabetes. Social factors such as poor quality of life, poor housing, overcrowding, and population explosion, under nutrition, lack of education, large families, early marriage and lack of awareness of causes of illness contribute to the occurrence and spread of tuberculosis. India being a developing country, it is one of the leading causes of mortality.

Pulmonary TB can be categorized into primary and post primary. Primary TB results from initial infection with tubercle bacilli. It is due to first-time exposure to mycobacterium bacilli. In TB-endemic regions, primary pulmonary TB often begins early in childhood with the inhalation of the tubercle bacillus however, it may be seen in adult, especially in developed countries, where the incidence of primary TB in adult has been reported to be $23 \%-34 \%{ }^{4}$. The site of primary $\mathrm{TB}$ in the lung reflects area of greatest ventilation, the most common sites are middle lobe, the lower lobe, or anterior segment of an upper lobe ${ }^{5}$ and associated with mediastinal lymphadenopathy. These lung lesions healed spontaneously and may be evident as a calcified nodule (ghon lesion). The lesion if progresses may produce pleural effusion, tuberculous empyema, acute cavitation with mediastinal lymphadenopathy. It may even progress to segmental or lobar collapse and may developed obstructive emphysema and bronchiectasis. In immune compromised individuals, it may develop miliary tuberculosis and tubercular meningitis.

Tuberculosis of lung though most frequent, may involve any organ in the body. Timely diagnosis is most important as delayed treatment is associated with severe morbidity. ${ }^{6}$ Post primary disease is due to reactivation and reinfection with the tubercle bacillus. ${ }^{6}$ It usually localised to apical and posterior segment of upper lobes and superior segment of lower lobes. In immunocompetent host, upper lobe cavitary lesion is typical and lower zone disease, adenopathy and effusion are hallmark of tuberculosis in immune-compromised host. ${ }^{7}$ In primary pulmonary TB, early diagnosis is very important for effective treatment. Though Chest X-Ray is done for parenchymal disease, Computed tomography (CT) is more sensitive in detecting lymphadenopathy. In post primary TB, CT is method of choice for detecting early bronchogenic spread. CT is more sensitive than X-Ray for characterisation of disease activity. ${ }^{8}$
Pulmonary TB may manifest as active or latent forms. Active pulmonary TB can be primary, post primary, developing after a long period of latent infection. Commonest CT findings in active disease are ill defined nodules, tree in bud pattern, consolidation, cavitation and ground glass opacities ${ }^{9}$ Primary TB manifests as lymphadenopathy, pulmonary consolidation and pleural effusion. Post primary pulmonary TB manifests as cavities, consolidation and centrilobular nodules. Miliary TB is most commonly seen in immune compromised patients. In 2-6\% of primary TB, the lymph node and haematogenous dissemination of massive number of viable organisms results in clinical and radiological evidence of miliary TB. Miliary TB can occur in post primary TB when the host's defence mechanism is overwhelmed ${ }^{10}$. Latent TB is asymptomatic and can progress to post primary TB. Radiologically, incipient or latent TB manifest as a small infiltrate in upper lobe either in the apical or sub-apical areas. From this stage onwards, tuberculosis develops in phases. Each worsening, as a rule, is acute in character while regressive changes are slow and chronic ${ }^{11}$

Atypical mycobacterium infection/ pulmonary nontuberculous mycobacterial infection (NTMB) is caused by agents other than $M$ tuberculosis. Mycobacterium aviumintracellulare complex (MAC) and Mycobacterium kansasii are the main causative agents. These are free living saprophyte. Infection is usually acquired from the environment by inhalation or ingestion. Most exposure doesn't cause disease. Frail elderly, immunocompromised, a defect in local and systemic defences are at highest risk. It usually presents with non-tender unilateral cervical lymphadenopathy in a systemically well children ${ }^{12}$. Radiological pattern of lung infection is usually indistinguishable from reactivation or re infection tuberculosis. It usually affects middle lobe or lingual lobe with centrilobular nodules or bronchiectasis. Early diagnosis is important as treatment regime is completely different from pulmonary tuberculosis.

An early and accurate diagnosis of pulmonary TB should be established using chest X-ray, sputum microscopy, culture in both liquid and solid media, and nucleic acid amplification. Chest CT, histopathological examination of biopsy samples, and new molecular diagnostic test can be used for earlier and improved diagnoses, especially in patients with smearnegative pulmonary TB or clinically diagnosed TB and drug resistant $\mathrm{TB}^{13}$. CT thorax is used mainly in diagnosis and follows up of TB. Sputum examination and culture takes many days and even weeks resulting delayed in detecting the diseases further delays in isolation the patient. Imaging played an important role in diagnosis and detection of TB. Chest x-ray is primary modality for diagnosis. CT is next investigation of choice in equivocal x-ray. Multidetector CT has important role in diagnosis of TB.

CT of chest can diagnose many diseases involving the chest wall, mediastinum, pleura, pulmonary arteries and lung parenchyma $^{14}$ the cause of unexplained cough, chest pain, fever and other chest symptoms. Various lung diseases like tuberculosis, pneumonias, benign and malignant diseases, interstitial lung diseases, chronic lung diseases, emphysema, bronchiectasis, congenital anomalies, pleural diseases and chest injuries etc. can be detected by CT scan of chest. CT scanning is fast, painless, noninvasive and accurate. It is very effective for patients who have trouble in holding the breath as it is very fast and painless. Another advantage of CT is its 
ability to image bone, soft tissue and blood vessel all at the same time. CT scanning can provide very detailed images of lung, bone and blood vessel unlike conventional X-ray. Owing to its high speed and better resolution, CT is the modality of choice for evaluation of TB. It also provides real time imaging for guiding invasive procedure for needle biopsy and needle aspiration in thorax. Chest radiography remains the first imaging technique in evaluation of thoracic tuberculosis. CT and High-Resolution CT (HRCT) may be needed for further evaluation of known or suspected TB in thorax. ${ }^{10}$

As pulmonary TB is a public health problem, prompt and early diagnosis of TB is a priority for TB control to prevent further spread. Diagnosis of TB is by chest X-Ray, sputum microscopy, culture and nucleic acid amplification. Chest XRay is not very specific for pulmonary TB as TB can present with atypical radiological findings. Many cases of pulmonary TB can be missed thus it may delay the treatment protocol. Sputum examination for acid fast bacilli is rapid and fast but sensitivity is very low. Again, sputum culture which is very accurate and highest sensitivity for acid fast bacilli takes around 4 to 8 weeks. Molecular and non-molecular assay are also developed for early detection of pulmonary TB. Pulmonary TB refers to any bacteriologically confirmed or clinically diagnosed case of TB that involves the lung parenchyma or tracheobronchial tree based on revised previous standard case definition for TB by World health organisation in 2013

Clinical sign and symptom of pulmonary TB is cough lasting for two weeks or more or unexplained chronic fever or weight loss should be investigated for pulmonary TB. Chest XRay is the first investigation. Chest X-Ray is useful but not specific. It may be even normal when the disease is present. CT can define local area of subpleural nodule or cavitation that is undetectable on routine chest radiograph ${ }^{15}$. Post primary TB usually present as cavitary lesion or patchy poorly defined consolidation in apical and posterior segment of upper lobe and superior segment of lower lobe. These lesions may not be accurately diagnosed by Chest-X-Ray. Although Chest-X-Ray is first investigation of choice for pulmonary TB, CT chest is required for detection of lesion that is overlooked on chest $\mathrm{X}$ ray and to evaluate disease progress and to detect complications. CT and HRCT are useful in detecting miliary and centrilobular nodules, ground glass opacities and air trapping. CT scan of thorax can detect and diagnose TB in patient with negative sputum and those without sputum production such as follow up cases of TB. It also helps in differential diagnosis of parenchymal lesions, assessing the disease activity and evaluating complications. It enables earlier and more accurate diagnosis of TB. Chest CT is an effective diagnostic method when chest X-Ray is normal or inconclusive. CT Chest can also give valuable information in bacterial activity. Active TB usually present as branching opacities, cavitary lesion and consolidation. Radiological finding in CT Chest is "tree-in-bud" pattern consisting of multiple small branching linear representing bronchogenic spread of disease with caseation necrosis in respiratory or terminal bronchioles ${ }^{11}$. These lesions when visualised with cavitation at apical and posterior segment of upper lobe or superior segment of lower lobe with appropriate clinical setting, specific diagnosis of pulmonary TB can be established. Centrilobular densities in and around the small airway and "tree-in-bud" appearance are the most characteristic CT features of disease activity. HRCT scanning clearly differentiated old fibrotic lesions from new active lesion and demonstrated early bronchogenic spread.16 Imaging play an important role in evaluation of chest TB patient and CT is more sensitive than chest X-ray in this regard. $^{7}$

The objective of this study was to evaluate the role of CT or HRCT in pulmonary TB in a series of cases.

\section{METHODS}

After taking ethical clearance, this cross-sectional study was conducted in 358 consecutive patients undergoing CT thorax over a period of six months for analysis of 6 months profile from 01-01-2016 up to 30-06-2016 at the department of Radiodiagnosis at JNIMS, Imphal. The cases referred to our department from Medicine and TB and Chest department for CT thorax presenting with cough, chest pain and difficulty in respiration. Patients irrespective of age, sex, religion, occupation, socio economic condition were included in the study. HRCT or CT thorax was done using 64 slice AQUILION Toshiba machine. For contrast enhanced CT, proper consent was taken. Proper history was taken regarding history of allergy, renal failure, diabetes, any surgery done, stent placement, heart catheterisation, open heart surgery, nephrectomy, born with one kidney or history of receiving chemotherapy etc. Renal functions were assessed. CT thorax was done with intravenous non-ionic contrast media if indicated. Nephrotoxicity of the contrast media used for CT remains a cause of concern, especially in patient with impaired renal function. ${ }^{12}$

For each patient following data like age, sex, consolidation, collapsed, cavitary lesions, pulmonary nodules, pleural effusion, pericardial effusion, mediastinal lymphadenopathy, fibrotic lesion, pleural calcification and bony chest wall involvement were collected and analysed for patients diagnosed with pulmonary tuberculosis. CT findings were analysed and correlated with clinical findings.

\section{Statistical Analysis}

All the data were entered in excel sheet and transferred into SPSS- 2 VERSION and then analysed by using descriptive statistic like mean, standard deviation and percentage. Chi square test was used. $\mathrm{P}$ value of $<0.05$ was taken as statistically significant.

\section{RESULTS}

In a series of 358 cases, $49.7 \%$ (178 cases) was pulmonary tuberculosis, $7.5 \%$ (27 nos.) Normal cases, pulmonary emphysema in 5.9\% (21 nos.) cases, 5.0\% (18 nos.)cases each of lung mass, traumatic cases, pneumothorax, 3.9\% (14 nos.) cases of idiopathic pulmonary fibrosis, $2.0 \%$ (7 nos.) cases each in pulmonary arterial hypertension, hypersensitivity pneumonitis, $1.7 \%$ (6 nos.) cases each in pulmonary oedema, sarcoidosis, cardiac diseases, $1.4 \%$ (5 nos.) cases each in empyema and idiopathic lung disease, $0.8 \%$ (3 nos.) Cases each in bronchopneumonia, mediastinal mass, lung metastasis, pleural mass, $0.3 \%$ ( 1 no.) case each in pericardial effusion, aortic aneurysm, bronchiectasis, lung abscess, 
solitary pulmonary nodule, costochondritis, oesophageal mass, foreign body obstruction were diagnosed.

\begin{tabular}{|c|c|c|}
\hline Cases & Number & Percentage \\
\hline Pulmonary tuberculosis & 178 & 49.7 \\
\hline Normal cases & 27 & 7.5 \\
\hline Pulmonary emphysema & 21 & 5.9 \\
\hline Lung mass & 18 & 5.0 \\
\hline Trauma cases & 18 & 5.0 \\
\hline Pneumothorax & 18 & 5.0 \\
\hline Idiopathic pulmonary fibrosis & 14 & 3.9 \\
\hline Pulmonary arterial hypertension & 7 & 2.0 \\
\hline Hypersensitivity pneumonitis & 7 & 2.0 \\
\hline Pulmonary oedema & 6 & 1.7 \\
\hline Sarcoidosis & 6 & 1.7 \\
\hline Cardiac diseases & 6 & 1.7 \\
\hline Empyema & 5 & 1.4 \\
\hline Idiopathic lung disease & 5 & 1.4 \\
\hline Broncho pneumonia & 4 & 1.1 \\
\hline Mediastinal mass & 3 & 0.8 \\
\hline Lung metastasis & 3 & 0.8 \\
\hline Pleural mass & 3 & 0.8 \\
\hline Pericardial effusion & 2 & 0.6 \\
\hline Aortic aneurysm & 1 & 0.3 \\
\hline Bronchiectasis & 1 & 0.3 \\
\hline Lung abscess & 1 & 0.3 \\
\hline Solitary pulmonary nodule & 1 & 0.3 \\
\hline Costochondritis & 1 & 0.3 \\
\hline Oesophageal mass & 1 & 0.3 \\
\hline Foreign body obstruction & 1 & 0.3 \\
\hline Total & 358 & 100 \\
\hline \multicolumn{3}{|c|}{ Table. 1 Distribution of Cases } \\
\hline
\end{tabular}

Among the patient diagnosed with pulmonary TB in 178 cases, there were 104 males (58.4\%) and 74 (41.6\%) were female. Mean age of all Pulmonary TB was (70.0 \pm 17.4$)$. Minimum age was 3 yrs. and maximum age was 98 yrs. Mean age distribution $(61.4 \pm 18.5)$ revealed highest in male where $(60.3 \pm 15.9)$ in female as shown in table 2 below.

\begin{tabular}{|c|c|c|c|c|}
\hline Cases & Number (\%) & Age (Mean \pm SD) & Min. Age & Max. Age \\
\hline Male & $104(58.4)$ & $61.4 \pm 18.5$ & 3 yrs. & 90 yrs. \\
\hline Female & $74(41.6)$ & $60.3 \pm 15.9$ & 18 yrs. & 98 yrs. \\
\hline Total & $\mathbf{1 7 8}(100)$ & $\mathbf{7 0 . 0} \pm 17.4$ & 3 yrs. & 98 yrs. \\
\hline \multicolumn{5}{|c|}{ Table 2. Sex \& Mean Age Distribution in Pulmonary TB } \\
\hline
\end{tabular}

In our study, incidence of pulmonary tuberculosis was highest (55.1\%) cases in the age group 61 yrs. and above. This was followed by $30.9 \%$ in the age group of $41-60$ years and $11.8 \%$ in the age group of 21-40 yrs. The minimum number of cases $(2.2 \%)$ was in the age group of $0-20$ years. Table 3 shows age and sex distribution whereas figure 2 shows percentage of cases in each group.

\begin{tabular}{|c|c|c|c|}
\hline Age in Years & Male (\%) & Female (\%) & No. of Cases (\%) \\
\hline $0-20$ & $3(2.9)$ & $1(1.4)$ & $4(2.2)$ \\
\hline $21-40$ & $13(11.8)$ & $8(10.8)$ & $21(11.8)$ \\
\hline $41-60$ & $27(30.9)$ & $28(37.8)$ & $55(30.9)$ \\
\hline 61 and above & $61(55.1)$ & $37(50.0)$ & $98(55.1)$ \\
\hline TOTAL & $104(100)$ & $74(100)$ & $178(100))$ \\
\hline \multicolumn{4}{|c|}{ Table 3. Age and Sex Distribution in Pulmonary TB } \\
\hline
\end{tabular}

Multiple pulmonary nodules in coronal section 4 depicts axial CT scan cavitary lesions. Based on site of involvement nature of findings, cases were diagnosed as TB with cavitary \& parenchymal lesions in 110 cases (61.8\%), TB with lung destruction \& cicatrisation in 23 cases $(12.9 \%)$, TB with bronchiectasis in 8 cases (4.5\%),TB with chronic tubercular empyema \& fibrothorax in 9 cases ( $5.1 \%$ ), TB with fungal infection in $11(6.2 \%)$,TB with trachea bronchial stenosis in 6 cases $(3.4 \%)$, miliary TB in $4(2.2 \%)$, TB with chest wall and vertebral involvement in $4(2.2 \%)$, TB with lung mass, pneumothorax and pericardial involvement in 1 cases each $(0.6 \%)$.

Over $60 \mathrm{yrs}$. of age $56.1 \%$ of the cases had TB with cavitary \& parenchymal lesions followed by TB with lung destruction \& cicatrisation in $17.3 \%$ of the cases. In individual up to 20 years of age $75 \%$ of cases had TB with cavitary \& parenchymal lesions and in $25 \%$ of the cases there were TB with chronic tubercular empyema \& fibrothorax. Incidence of TB in higher age groups were statistically significant $(p<0,05)$ (Table 4).

\begin{tabular}{|c|c|c|c|c|c|}
\hline \multirow[b]{2}{*}{ Type of Cases } & \multicolumn{4}{|c|}{ Number of Cases (\%) } & \multirow[b]{2}{*}{$\begin{array}{c}\text { Total } \\
(\%)\end{array}$} \\
\hline & $0-20$ & $21-40$ & $41-60$ & $\begin{array}{c}61 \& \\
\text { above }\end{array}$ & \\
\hline $\begin{array}{l}\text { TB with cavitary \& } \\
\text { parenchymal lesion }\end{array}$ & $3(75.0)$ & $\begin{array}{c}17( \\
(81.0) \\
\end{array}$ & $35(63.6)$ & $55(56.1)$ & $\begin{array}{c}110 \\
(61.7)\end{array}$ \\
\hline $\begin{array}{c}\text { TB with lung destruction \& } \\
\text { cicatrization }\end{array}$ & - & - & $6(10.9)$ & 17 (17.3) & $23(12.9)$ \\
\hline TB with bronchiectasis & - & - & $1(1.8)$ & $7(7.1)$ & $8(4.5)$ \\
\hline $\begin{array}{l}\text { TB with chronic tubercular } \\
\text { empyema \& fibrothorax }\end{array}$ & $1(25.0)$ & $1(4.8)$ & $4(7.3)$ & $3(3.1)$ & $9(5.1)$ \\
\hline TB with fungal infection & - & $1(4.8)$ & $3(5.5))$ & $7(7.1)$ & $11(6.2)$ \\
\hline $\begin{array}{c}\text { TB with trachea bronchial } \\
\text { stenosis }\end{array}$ & - & - & $2(3.6)$ & $4(4.1)$ & $6(3.4)$ \\
\hline Miliary TB & - & $2(9.5)$ & $2(3.6)$ & - & $4((2.2)$ \\
\hline $\begin{array}{c}\text { TB with chest wall \& vertebral } \\
\text { involvement }\end{array}$ & - & - & - & $4(4.1)$ & $4(2.2)$ \\
\hline TB with lung mass & - & - & $1(1.8)$ & - & $1(0.6)$ \\
\hline TB with pneumothorax & - & - & & $1(1.0)$ & $1(0.6)$ \\
\hline $\begin{array}{c}\text { TB with pericardial } \\
\text { involvement }\end{array}$ & - & - & $1(1.8)$ & & $1(0.6)$ \\
\hline Total & $4(100)$ & $21(100)$ & $55(100)$ & $98(100)$ & $\begin{array}{c}178 \\
(100)\end{array}$ \\
\hline \multicolumn{6}{|c|}{ Table 4. Type of Pulmonary TB among Different Age Groups } \\
\hline 73.171 with 33d.f; $p=0.000$ & 05 & ificant & & & \\
\hline
\end{tabular}

\section{DISCUSSION}

In our retrospective study 178 out of 358 i.e. $49.7 \%$ were diagnosed as pulmonary TB. Among which 104 i.e. $58 \%$ were male and 74 i.e. $42 \%$ were female. Males were more affected than female. In virtually all countries, more male than female cases of tuberculosis was reported ${ }^{17}$. Our finding male-female ratio was 1.4:1. Compare to a study incidence of pulmonary TB in male is $58 \%$ and in female incidence is $41.1 \%{ }^{18}$. Cases diagnosed as pulmonary TB had history of chronic cough with sputum, chest pain, shortness of breath, haemoptysis, fever, weight loss etc. Classical clinical features of pulmonary TB include chronic cough, sputum production, appetite loss, weight loss, fever night sweats and haemoptysis.

Typical CT finding of active post primary tuberculosis are centrilobular branching nodules, lobular consolidation, cavitation and bronchial wall thickening ${ }^{19}$. Parenchymal \& cavitary lesions were found in 110 cases (61.7\%) in our study compare to a study where cavitary nodules and consolidation were found in $64 \%$ of cases. ${ }^{20}$ Early active pulmonary TB shows thick walled cavitary lesion usually within a consolidated nodule. Hallmark of post primary TB is cavitation. Lung destructions and cicatrization were found in 23 cases $(12.9 \%)$, bronchiectasis in 8 cases $(4.5 \%)$, chronic empyema and fibrothorax in 9 (5.1\%), tracheobronchial stenosis in $6(3.1 \%)$, miliary TB in $4(2.2 \%)$, chest wall and vertebral involvement in $4(2.2 \%)$, TB with lung mass, pneumothorax and pericardial involvement in 1 case each 
(0.6\%). Compare to a study, ${ }^{21}$ males were $52 \%$ and females were $47.29 \%$ which is comparable with our study. Cases of consolidation were $66.84 \%$ in sputum positive cases, lung atelectasis or collapse in $8.02 \%$, traction bronchiectasis in $16.4 \%$ slightly higher compare to our study.

Lung consolidations were found in $70 \%$ of primary $\mathrm{TB}$, cavities, the radiological hallmark of reactivation TB in 20 $40 \%$ caeses $^{22}$ Miliary TB is also found in $2-6 \%$ of primary TB. ${ }^{23}$ Pulmonary TB sequelae like cicatrization and lung destruction is common finding in post primary TB usually around $40 \%$ post primary $\mathrm{TB}^{23}$ manifested as atelectasis, hyperinflation, fibrotic bands, cavities and traction bronchiectasis. Bronchiectasis develops in $30 \%-60 \%$ active post primary TB and $71 \%-86 \%$ of inactive TB in HRCT. ${ }^{24}$ Trachea-bronchial stenosis is around $2 \%-4 \%$ compare to $3.1 \%$ in our findings. Tuberculous pleurisy develops into chronic tubercular empyema. Fibrothorax and diffuse pleural thickening indicates disease inactivity. Pneumothorax is found in 5\% of post primary cases slightly higher than our finding of $4.5 \%$. Chest wall involvement is seen as bone or costal cartilage destruction with associated soft tissue masses showing rim enhancement. TB spondylitis usually involve lower thoracic and upper lumbar spine as end plate erosions, sclerosis, reduced disc space with paravertebral abscess.

CT scan can provide more accurate information about the extent of disease and distribution by detecting presence of cavities and satellite lesions that cannot be visualised in chest $\mathrm{X}$-Ray. In most of the cases, active and inactive or latent can be determined based on CT findings. CT scan can evaluate the disease activity, can detect complications as well as sequelae. ${ }^{24}$ CT scan has its own limitations like radiation, needs to inject contrast for evaluating mediastinum in some cases etc. Despite its limitation, CT scan is very useful in diagnosing pulmonary TB. CT Scanning is more sensitive than chest radiography in depicting cavitation, rupture into the pleural space, mediastinal adenopathy. HRCT scans demonstrated early bronchogenic spread which helps in identifying active disease.

\section{CONCLUSIONS}

Pulmonary TB is an important public health issue, more so in a developing country like India. Despite many advances in both diagnosis and treatment, TB still remains one of the commonest causes of morbidity and mortality from any infectious cause in the world. Although the overall incidence and mortality rate for tuberculosis has decreased over the year, timely and accurate diagnosis of TB is essential for the health of the patient as well as the public. CT scan is valuable for establishing diagnosis and monitoring disease activity, detecting disease complications and exploration of unexplained lung disease. Chest $\mathrm{x}$-ray is still used for screening of TB, though it has its own limitation in diagnosing TB in sputum negative cases. CT can identify or confirm the presence of findings in patients with clinical sign of active disease with normal chest $x$-ray. ${ }^{24}$

Though chest $\mathrm{x}$-ray is the first imaging technique of choice for diagnosis of pulmonary TB, the main role of HRCT chest is in diagnosing pulmonary TB in highly suspected TB with pulmonary infiltrates of unknown origin with negative sputum smear. It can segregate high risk patient in those suspected TB cases $^{25}$. HRCT is better than plain x-ray in detecting extent of pulmonary $\mathrm{TB}$, in subtle areas of consolidation, cavitation, bronchogenic and miliary spread. HRCT chest is recommended in cases where chest $\mathrm{x}$-ray findings are normal or inconclusive in clinically suspected cases for confirmation of diagnosis and determination of disease activity. Even in TB outbreaks preventing disease transmission by detecting active cases is important. Inclusion of HRCT over and above the outbreak investigation of TB may be helpful in differentiating active TB from latent TB. ${ }^{26}$ CT thorax is a must for differentiating different types and manifestations of pulmonary TB as well as differentiating it from nontuberculous mycobacterium. CT especially HRCT is superior to chest radiography in assessing tuberculosis activity. However, for accurate diagnosis, correlated study with sputum examination is a must.

\section{REFERENCES}

[1] Jeong YJ, Lee KS. Pulmonary tuberculosis: up-to-date imaging and management. Am J Roentgenol 2008;191 (3):834-44.

[2] Foulds J, O'Brien R. New tools for the diagnosis of tuberculosis: the perspective of developing countries. Int J Tuberc Lung Dis 1998;2 (10):778-83.

[3] Ashu SB, Ankur G, Randeep G, et al. Chest tuberculosis: Radiological review and imaging recommendations. Indian J Radiol Imaging 2015;25 (3):213-25.

[4] Miller WT, Miller WT Jr. Tuberculosis in the normal host: radiological findings. Semin Roentgenol 1993;28 (2):10918.

[5] Lee KS, Im JG. CT in adults with tuberculosis of chest: characteristic findings \& role in management. Am J Rheontgenol 1995;164 (6):1361-7.

[6] Joshua B, Christopher JW, Gillian B, et al. Tuberculosis: a radiologic review. Radiographics 2007;27 (5):1255-73.

[7] Anna R, Frank H, Michael ST, et al. Radiographic appearance of pulmonary tuberculosis: Dogma disproved. Am J Roentgenol 2015;204 (5):974-8.

[8] Evangelia S, Alimuddin Z, Jamshed B. Imaging in tuberculosis. International Journal of Infectious Diseases 2015;32:87-93.

[9] Lee YJ, Lee KS, Jung KJ, et al. Pulmonary tuberculosis; CT and pathological correlation. J Comput Assist Tomogr 2000;24 (5):691-8.

[10] Lee KS, Song SK, Lim HT, et al. Adult-onset pulmonary tuberculosis: findings on chest radiographs and CT scans. AM J Roentgenol 1993;160 (4):753-8.

[11] Behera G, Dei KL, Mania RN et al. Acute radiological change in adult pulmonary tuberculosis. Ind $\mathrm{J}$ Tub 2002;49:157-8.

[12] Surendran T, Louise MM, Chris H, et al. Atypical mycobacterial infection in children: the case for early diagnosis. Ulster Med J 2006;75 (3):192-4.

[13] Yon RJ. Diagnosis of pulmonary tuberculosis: recent advances and diagnostic algorithms. Tuberc Respir Dis (Seoul) 2015;78 (2):64-71.

[14] Camila PP, Rahul R, Michael BA. When dose chest CT require contrast study. Cleveland Clinic Journal of Medicine 2016;83 (6):423-6. 
[15] Arun CN, Kasra R, Xiao S, et al. Pulmonary tuberculosis: role of radiology in diagnosis and management. RSNA Radiographic 2017;37 (1):52-72.

[16] Hatipoglu ON, Osma E, Mnisali M, et al, High resolution computed tomographic findings in pulmonary tuberculosis. Thorax 1996;51 (4):397-402.

[17] Borgdorff MW, Nagelkerke NJ, Dye C, et al. Gender and tuberculosis: a comparison of prevalence survey with notification of data to explore sex differences in case detection. Int J Tuberc Lung Dis 2000;4 (2):123-32.

[18] Jimennez-Corona M-E, Garcia-Garcia L, DeReimer K, et al. Gender differentials of pulmonary tuberculosis transmission and reactivation in an endemic area. Thorax 2006;61 (4):348-53.

[19] Soujanya B, Chhaya B, Dharita S. Role of HRCT in predicting disease activity of pulmonary tuberculosis. Gujarat Medical Journal 2014;69:91-5.

[20] Raniga S, Parikh N, Arora A, et al. Is HRCT reliable in determining disease activity in pulmonary tuberculosis? Int J Radiol Imag 2006;16 (2):221-8.

[21] Pepper T, Joseph P, Mwenya C, et al. Normal chest radiography in pulmonary tuberculosis: implications for obtaining respiratory specimen culture. Int J Tuberc Lung Dis 2008;12 (4):397-403.
[22] Hua H, Pu-Xuan L. Paving-stone CT finding in a pulmonary tuberculosis patient. Quant Imaging Med Surg 2013;3 (5):282-3.

[23] Shivraj MI, Swapnil SI, Prashant GP, et al. Importance of HRCT for prediction of disease activity in pulmonary tuberculosis. MedPulse - International Medical Journal 2017;4 (6):828-30.

[24] Drusty MK, Deepak KR. Role of HRCT in diagnosing disease activity in pulmonary tuberculosis. International Journal of Contemporary Medical Research 2017;4 (8):1724-7.

[25] Kim HY, Song KS, Goo JM, et al. Thoracic sequelae and complication of tuberculosis. Radiographics 2001;21 (4):839-58.

[26] Boushab MB, Ould-Bahiya SY, Mamoudou S, et al. Contribution of computed tomography in the diagnosis of pulmonary tuberculosis at Kiffa regional hospital, Assaba. International Journal of Pulmonary \& Respiratory Sciences 2018;3 (5):1-5. 